\title{
Novel Systems Modeling Methodology in Comparative Microbial Metabolomics: Identifying Key Enzymes and Metabolites Implicated in Autism Spectrum Disorders
}

\section{Colin Heberling ${ }^{1,2, *}$ and Prasad Dhurjati ${ }^{2}$}

1 Department of Biotechnology, Johns Hopkins University, Rockville, MD 20850, USA

2 Department of Chemical and Biomolecular Engineering, University of Delaware, Newark, DE 19716, USA; E-Mail: dhurjati@udel.edu

* Author to whom correspondence should be addressed; E-Mail: cheb@udel.edu; Tel.: +1-302-690-1003.

Academic Editor: Merlin G. Butler

Received: 14 January 2015 / Accepted: 24 March 2015 / Published: 22 April 2015

\begin{abstract}
Autism spectrum disorders are a group of mental illnesses highly correlated with gastrointestinal dysfunction. Recent studies have shown that there may be one or more microbial "fingerprints" in terms of the composition characterizing individuals with autism, which could be used for diagnostic purposes. This paper proposes a computational approach whereby metagenomes characteristic of "healthy" and autistic individuals are artificially constructed via genomic information, analyzed for the enzymes coded within, and then these enzymes are compared in detail. This is a text mining application. A custom-designed online application was built and used for the comparative metabolomics study and made publically available. Several of the enzyme-catalyzing reactions involved with the amino acid glutamate were curiously missing from the "autism" microbiome and were coded within almost every organism included in the "control" microbiome. Interestingly, there exists a leading hypothesis regarding autism and glutamate involving a neurological excitation/inhibition imbalance; but the association with this study is unclear. The results included data on the transsulfuration and transmethylation pathways, involved with oxidative stress, also of importance to autism. The results from this study are in alignment with leading hypotheses in the field, which is impressive, considering the purely in silico nature of this study. The present study provides new insight into the complex metabolic interactions underlying autism, and this novel methodology has potential to be useful for developing new hypotheses. However, limitations include sparse genome data availability and conflicting
\end{abstract}


literature experimental data. We believe our software tool and methodology has potential for having great utility as data become more available, comprehensive and reliable.

Keywords: autism; metabolomics; comparative; microbial; computational; bioinformatics; biomarkers; gut; gastrointestinal; microbiome

\section{Introduction}

Autism spectrum disorders are a category of mental illnesses characterized by social cognitive impairments and stereotyped behaviors [1]. Autistic individuals often have trouble fitting into society and put a financial burden on their families, lifelong. Autism diagnosis has been steadily on the rise in the past decade or so, affecting one in every 88 children according to a source in 2012 [1] and one in every 68 children from current data (2015) from the Centers for Disease Control and Protection [2]. There are most likely many adults suffering from autism that have never been diagnosed. This is because there is as of yet no clear method to diagnose autism; diagnosis is purely based on making qualitative observations of an individual. This is why there is a pressing need to find reliable methods for autism diagnosis and for treatment, as well.

Recent research suggests that individuals with autism often suffer from gastrointestinal dysfunction, as well $[3,4]$. Rather than focusing on the illness by way of human genetics, many scientists are now exploring the impact of microbial genetics. There exists a full isolated ecosystem of microbial lifeforms that inhabit the human gastrointestinal tract. These microbes have a profound impact on the health and disease of the human host and in general have a symbiotic co-existence with the host [5]. Microbial species or strain composition is believed to largely contribute to homeostasis or abnormality in humans. While each person's microbial "fingerprint" is unique, there are specific patterns seen in those that are healthy and those that have specific illnesses [6,7]. Remarkably, autism has been correlated with the overgrowth of certain types of bacteria, such as certain species of Clostridia [8,9] and Desulfovibrio [9,10], and there is some preliminary evidence that Sutterella may be implicated, as well [11]. Data from the pyrosequencing study by Finegold et al. [9] suggested that several other organisms may be implicated in autism, as well, but these results were less significant than those implicating Clostridia and Desulfovibrio.

Nonetheless, one organism, Akkermansia, was chosen from among these organisms to be included in the study, to see how much of an impact these less significant organisms might have. Akkermansia is part of the Verrucomicrobia phylum. According to a study by Williams et al. [12] where live intestinal biopsies were taken to measure bacterial composition, Verrucomicrobia made up approximately $1 \%$ of the total bacterial composition in individuals with autism and only $0.5 \%$ in healthy age-matched controls. This was another leading factor for the decision to include Akkermansia in the present study.

As one might expect, as bacterial composition has an effect on health and disease, the molecular metabolites that these microbes produce are the "tools" that carry out these biological changes. For example, individuals with autism have been cited as having sulfur metabolic deficiencies, to suffer from elevated oxidative stress and to have trouble detoxifying xenobiotic compounds and heavy metals [13-15]. It is therefore important to analyze the metabolome of these individuals and that of healthy individuals (for control) in order to identify useful biomarkers and perhaps even gain a greater 
understanding of the disorder. Knowledge may be gained about other related illnesses, as well, such as others related to gastrointestinal dysfunction.

Metagenomics is the study of the genetic content contained in whole microbial ecosystems isolated from natural environments $[16,17]$. In contrast, the more traditional genomics is the study of only DNA from a single, isolated species or strain of organism. Metagenomics therefore brings with it new challenges in bioinformatics. This is because before regular genomics can be applied to each organism, the key organisms need to be identified by aligning sample DNA reads to reference sequences contained in curated databases [18]. Of course, the environment that this study focuses on is the human gut microbiome. This study does not use the conventional methods of metagenomics, but rather simplifies the problem by constructing artificial metagenomes based on genomes of organisms already known to be contained in the target environment. There are numerous pieces of literature already describing the composition of microbes contained in autistic individuals and "healthy" individuals. The present study constructs a metagenomics "model", rather than conducting a pure metagenomics study.

\section{Methods}

The literature suggests that there are distinct microbiomes characteristic of a "healthy" individual and that of an autistic individual [4]. Therefore, the first step was to choose the microorganisms to represent these microbiomes. It was the intended goal to be as comprehensive as possible with this step, so that all possible enzymes and metabolites that are possible to exist in urinary or stool samples are covered. Choosing the correct microorganisms can be tricky, as sometimes there are conflicting data or interpretations of data in different literature [19]. Another quandary is whether to include the more common organisms in both microbiomes studied or to only include the "problem" organisms; that is, the microorganisms thought to cause the main differences between the two microbiomes. In the latter case, the autistic microbiome could be represented with just a few species of microbe. The end goal is to find the key enzymes and metabolites expressed that are entirely different in the autistic individual. Of course, autism is a complex disorder with multiple different pathways for pathogenesis, so the autistic microbiome chosen will try to represent all cases of autism arising from gut microbiological origin.

Only those microorganisms thought to be represented in significantly higher amounts (proportionally) in the guts of autistic individuals were chosen for the autistic microbiome, with the core microorganisms representative of a healthy human gut for the control microbiome. However, there is still no consensus on what defines a typical "healthy" gut microbiome, so the "control" microbiome was chosen based on a few key steps. First, those microbes that have been reported to be in decreased quantities in autism were included in the "control" microbiome $[9,12,20]$. Next, those reported to have no change in composition were included. Finally, other organisms that are normally found in the human gut, but that had very little discussion in related autism literature were included in the "control" microbiome. To facilitate this final step, we have unpublished work where the MEtaGenome Analyzer (MEGAN software) [21,22] was used to analyze three sets of metagenomic data from the Human Microbiome Project's collection of human stool samples [6,23]. These design criteria may seem confusing at first if we take into consideration that those microorganisms that are decreased in autism are nonetheless still implicated in autism, just like those organisms that are found in increased amounts. However, our design considerations are predicated on the assumption that those organisms that are found in decreased 
numbers in autism are in fact usually beneficial for the host in otherwise "healthy" microbiomes. There is at least some evidence for two such cases: Lactobacillus species and Bifidobacterium species [24]. We can therefore justify stratifying gut microbes into two groupings based on a simple binary distinction: those microbes that are found in increased numbers in autism and those microbes that are not found in increased numbers in autism. Comparing these two groups should reveal key differences in expressed enzymes. Thus, finding enzymes that are expressed in the "autism" group and not in the "control" group may identify metabolites indicative only of our "problem" organisms and thus may serve as potential biomarkers for autism. On the other hand, finding enzymes that are expressed by the "control" group and not the "autism" group may also identify key biomarkers, except in this case, clinicians may be able to look for decreased quantities of such molecules instead.

Enzyme Commission (EC) numbers were used for the comparative analysis. EC numbers are a standardized way to identify enzymes, much like CAS numbers (Chemical Abstracts Service) for common chemicals. GenBank files for each microbe were downloaded from the National Center for Biotechnology Information (NCBI) [25]. The limitations of this study occurred at this stage. Not all microbes known have a completely sequenced genome uploaded to NCBI, and even less still have their protein products annotated with EC numbers. This greatly limited which organisms could be used in the study. The following list organizes microorganisms into three categories: those included in the first software query, those additional organisms that were able to be included in Query 2 after de novo EC number annotations were made (more detail later) and those organisms that were desired to be included, but in the end were not.

The usual approach for metagenomics presents significant bottlenecks for analysis and data storage. This is why the idea came about that there may be another approach that is more efficient in very specific circumstances. Instead of using metagenomic data, this study attempts to construct an artificial metagenome by carefully choosing the microorganisms to include in the model and using the curated database of GenBank files to analyze the full spectra of enzymes coded in the genomic DNA of these organisms. This study is differentiated from a proteomics study, because we are only interested in proteins that have been assigned a standardized Enzyme Commission (EC) number, so that there is no ambiguity between gene products. Two enzymes from two different organisms may have the same EC number, but slightly different amino acid sequences or slightly different protein names in the GenBank annotation files. Using EC numbers will allow automated programs to match identical enzymes correctly. The enzymes are correlated with their associated chemical reactions and the implicated metabolites. This method of intentionally leaving out some information and looking at the bigger picture on more of a systems level could be classified as systems biology [26].

A personal computer was used for all programming and analysis. A simple, personally-designed online application was used for all analysis. This software can be found at the web link [27]. Full instructions on how to use the software can be found in a link to the readme file on the main program interface linked above. The software utilizes a relational database system using MySQL to store and query information from the GenBank annotation files from NCBI and information from an EC number database [28]. The EC number data file was parsed for inserting data into a personally-designed database table. The following example (Figure 1) shows the type of information contained in the data file that was parsed for EC numbers. 


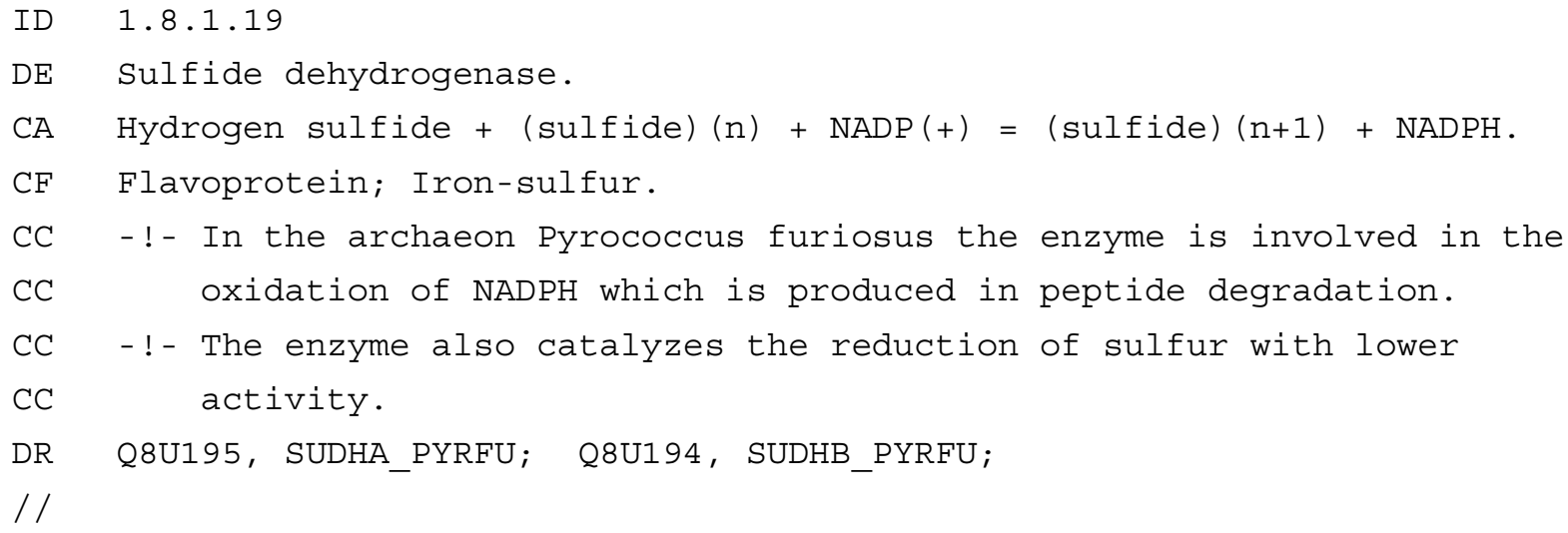

Figure 1. Example data extracted from The ENZYME Database in 2000, Bairoch [28].

The web application allows its user to choose two groups of microbiomes for metabolomics comparison. The program was designed with the intent to be used for any microbial metabolomics study between two microbiomes, two small alterations of the same microbiome or even simply between two single organisms. The application allows the user to upload a new genome file in GenBank format. Initially, the application could only accept GenBank files with EC number annotations present, but now, there does not need to be any EC number annotations in the original file. However, inserting EC number annotations with automation has certain limitations. Database queries fall into three categories for each comparison: no matches, one unique match or more than one match. In the event that there is more than one match, manual curation will be necessary. In the meantime, the program instead prints the first three matches to the GenBank file and makes a designation within the file and within the associated database that says that the information may be less reliable than unique matches or the data that was already annotated in the original file.

The application returns output directly to the screen within the web browser. The output consists of a data table, where each row contains columns showing the GI accession number (from NCBI) of the organism that the data come from, the organism's common name, an EC number, the predominant name associated with that EC number, the associated biochemistry, a "reliability factor" and a keyword describing which microbiome the data are associated with (default "Microbiome 1" and "Microbiome 2", or custom names supplied by the user on the form page). The table is broken up into three major sections: those enzymes that are identical between the two microbiomes and those enzymes that are unique for each microbiome. For this study (and likely others that might benefit from this software package), the sub-tables showing the unique results are most useful. An example partial result (Figure 2) might be as follows. 
Organisms in Microbiome1:

187426706 Akkermansia muciniphila ATCC BAA-835

Organisms in Microbiome2:

149935097 Bacteroides vulgatus ATCC 8482

291526581 Eubacterium rectale DSM 17629

Received search term query ".

Similar coded enzymes: 619 match(es).

\begin{tabular}{|c|c|c|c|c|c|c|}
\hline $\begin{array}{l}\text { GI } \\
\text { Accession }\end{array}$ & $\begin{array}{l}\text { Organism } \\
\text { Name }\end{array}$ & EC_number & Enzyme Name & Biochemistry & Reliability & $\begin{array}{l}\text { Microbiome } \\
\text { Name }\end{array}$ \\
\hline 187426706 & $\begin{array}{l}\text { Akkermansia } \\
\text { muciniphila } \\
\text { ATCC BAA- } \\
835\end{array}$ & 1.3 .1 .12 & $\begin{array}{l}\text { Prephenate } \\
\text { dehydrogenase. }\end{array}$ & $\begin{array}{l}\text { Prephenate }+\mathrm{NAD}(+)=4- \\
\text { hydroxyphenylpyruvate }+\mathrm{CO}(2)+ \\
\text { NADH. }\end{array}$ & 0 & Microbiome1 \\
\hline 291526581 & $\begin{array}{l}\text { Eubacterium } \\
\text { rectale DSM } \\
17629\end{array}$ & 1.3 .1 .12 & $\begin{array}{l}\text { Prephenate } \\
\text { dehydrogenase. }\end{array}$ & $\begin{array}{l}\text { Prephenate }+\mathrm{NAD}(+)=4- \\
\text { hydroxyphenylpyruvate }+\mathrm{CO}(2)+ \\
\mathrm{NADH} .\end{array}$ & 1 & Microbiome2 \\
\hline 187426706 & $\begin{array}{l}\text { Akkermansia } \\
\text { muciniphila } \\
\text { ATCC BAA- } \\
835\end{array}$ & 6.5.1.2 & $\begin{array}{l}\text { DNA ligase } \\
(\mathrm{NAD}(+))\end{array}$ & $\begin{array}{l}\mathrm{NAD}(+)+(\text { deoxyribonucleotide })(\mathrm{n}) \\
+(\text { deoxyribonucleotide })(\mathrm{m})=\mathrm{AMP}+ \\
\text { beta-nicotinamide } \mathrm{D}-\text { ribonucleotide }+ \\
(\text { deoxyribonucleotide })(\mathrm{n}+\mathrm{m}) .\end{array}$ & 1 & Microbiome1 \\
\hline 291526581 & $\begin{array}{l}\text { Eubacterium } \\
\text { rectale DSM } \\
17629\end{array}$ & 6.5.1.2 & $\begin{array}{l}\text { DNA ligase } \\
(\mathrm{NAD}(+))\end{array}$ & $\begin{array}{l}\mathrm{NAD}(+)+(\text { deoxyribonucleotide })(\mathrm{n}) \\
+(\text { deoxyribonucleotide })(\mathrm{m})=\mathrm{AMP}+ \\
\text { beta-nicotinamide D-ribonucleotide }+ \\
(\text { deoxyribonucleotide })(\mathrm{n}+\mathrm{m}) .\end{array}$ & 1 & Microbiome2 \\
\hline
\end{tabular}

Figure 2. Example extract from the original software results page.

In Figure 2, GI accession is the unique identifier for that GenBank record within NCBI, and the reliability column differentiates between new annotations with multiple EC number matches (reliability $=0$ ) and new annotations with unique matches and old annotations (reliability $=1$ ).

Three queries total were made with the web application. The first involved the organisms from the top section of Table 1. The second added the five organisms from the middle section of Table 1. In the third query, we tried to filter our results to just the organisms that are thought to be most important in each microbiome. These microbiomes consisted of those in Table 2. Table 1 is also available in the Supplemental Material as Table S1. 
Table 1. Microorganisms chosen for the comparative metabolomics study. Organisms included in Query 2 had de novo Enzyme Commission (EC) number annotations.

\begin{tabular}{|l|ll|}
\hline Query & "Healthy" Microbiome & "Autism" Microbiome \\
\hline Included in Query 1 & Bacteroides fragilis & Desulfovibrio desulfuricans \\
& Bifidobacterium longum & Clostridium perfringens \\
& Ruminococcus bromii & Clostridium difficile \\
& Roseburia intestinalis & Akkermansia muciniphila \\
& Faecalibacterium prausnitzii & \\
& Eubacterium rectale & \\
& Alistipes putredinis & \\
& Alistipes shahii & \\
& Weissella koreensis & \\
& Prevotella ruminicola & \\
& Odoribacter splanchnicus & \\
& Methanobrevibacter smithii & \\
& Clostridium leptum & \\
& Lactobacillus acidophilus & \\
\hline Additional organisms included & Bacteroides thetaiotaomicron & Sutterella wadsworthensis \\
& Bacteroides caccae & Clostridium bolteae \\
& & Bacteroides vulgatus \\
\hline in Query 2 & Bacteroides finegoldii & Desulfovibrio piger \\
& Bacteroides ovatus & Desulfovibrio intestinalis \\
& Bacteroides uniformis & Clostridium butyricum \\
& Bifidobacterium adolescentis & Clostridium paraputrificum \\
& Bifidobacterium pseudolongum & Clostridium subterminale \\
& Eubacterium siraeum & Clostridium tertium \\
& Dorea & Clostridium bifermentans \\
& Veillonella & Clostridium glycolicum \\
& Turicibacter & Bacteroides stercoris \\
& Barnesiella intestinihominis & Parabacteroides distasonis \\
& Odoribacter laneus & Parabacteroides merdae \\
& Dialister invisus & Paraprevotella xylaniphila \\
& & Eubacterium eligens \\
& & Prevotella oulorum \\
\hline
\end{tabular}

Table 2. Selected organisms for Query 3 of the Metabolomics Software.

\begin{tabular}{cc}
\hline & Organisms in Control: \\
\hline 392623967 & Bacteroides caccae CL03T12C61 \\
60495220 & Bacteroides fragilis ATCC 25285 = NCTC 93 \\
29342100 & Bacteroides thetaiotaomicron VPI-5482 \\
666001751 & Bifidobacterium longum BXY01 \\
488447870 & Lactobacillus acidophilus La-14 \\
148552872 & Methanobrevibacter smithii ATCC 35061; PS; DSMZ \\
\hline
\end{tabular}


Table 2. Cont.

\begin{tabular}{lc}
\hline & Organisms in Autism: \\
\hline 149935097 & Bacteroides vulgatus ATCC 8482 \\
480704622 & Clostridium bolteae 90A9 \\
110676061 & Clostridium perfringens ATCC 13124 \\
219869941 & Desulfovibrio desulfuricans ATCC 27774 \\
115252745 & Peptoclostridium difficile 630 \\
512689910 & Sutterella wadsworthensis HGA0223 \\
\hline
\end{tabular}

The organisms chosen for each query were input into the program and the results copied and pasted into an Excel file (available as the Supplemental Material, Tables S2-S7: each query is separated into two worksheets, one for complete raw data, and one for filtered data of interest). The "unique" results were looked through manually and considered on a case by case basis, with literature data in mind. Special attention was paid to those enzymes and metabolites found in the methionine and cysteine metabolic pathways and those involved with oxidative stress [13] and amino acid metabolism [29-31].

\section{Results}

The full results will not be reproduced here, but can easily be reproduced using the online software and the methods detailed above. The full results for each query are available as the Supplemental Material, Tables S2, S4, and S6. Additionally, the full results have been filtered for the most meaningful results and pasted within the Supplemental Material, Tables S3, S5, and S7. The first query resulted in the most meaningful results. It had 19,959 identical enzyme matches between the two microbiomes tested, 834 unique enzymes for the "control" microbiome and 161 unique enzymes for the "autism" microbiome. Gene copies are included in this count, so these numbers are a bit inflated. The identical enzymes are meaningless for this study, so we direct our attention to the tables of "unique" enzymes. Any meaningful results must be found manually. Table 3 below shows the differences in match statistics between each query. The majority of the results found with Query 1 were also found in Queries 2 and 3, with possibly a few minor differences. Judging by the quantity of the results, these minor differences were assumed to be near negligible. Therefore, when comparing Queries 1 and 2, we can come up with an approximate difference in the number of unique results by subtracting the statistics. Therefore, for instance, Query 2 only had 19 new results unique to the "control" microbiome and 34 new results unique to the "autism" microbiome, compared to the results of Query 1. Thus, one can see that Queries 2 and 3 would have less novel results overall than Query 1, given that Query 1 was conducted first.

Table 3. Comparing match statistics between web application queries.

\begin{tabular}{cccc}
\hline Query & Similar Enzymes & Control Unique & Autism Unique \\
\hline 1 & 19,959 & 834 & 161 \\
2 & 41,035 & 853 & 195 \\
3 & 14,451 & 356 & 387 \\
\hline
\end{tabular}


From an experimental study on metabolic biomarkers in autistic individuals [29], we know that many amino acids are found in significantly lower abundance vs. controls, including glycine, serine, threonine, alanine, histidine, glutamine, glutamate and the organic acid, taurine. Antioxidants (especially glutathione) are also in much lower abundance. Another study [30] cites reduced glutathione as being in much lower abundance and another type of antioxidant, thioredoxins, as being in much higher abundance. James et al. [13] claims that there is a metabolic bottleneck in many autistic individuals, where the conversion of methionine to cysteine is inhibited, leading to diminished glutathione formation. This information was kept in mind when analyzing the data. The relevance of these findings will be discussed in more detail in the Discussion Section.

In Tables 4-11 we present the most meaningful results obtained from the data mining. The majority of the results come from the first web application query. First, for the enzymes unique to the "control" microbiome, we present enzymes indicative of glutamate metabolism. Some of the entries below contain notation, such as “ $\times 3$ " after an organism name. This denotes that that organism has that number of gene copies coding for that particular enzyme (the first table with this notation is Table 6 below).

Table 4. Acetylornithine transaminase expression ("control” unique).

\begin{tabular}{lll}
\hline 2.6.1.11 & Acetylornithine Transaminase & $\begin{array}{l}\boldsymbol{N} \text { (2)-Acetyl-L-ornithine + 2-Oxoglutarate }= \\
\text { N-Acetyl-L-glutamate5-semialdehyde + L-Glutamate }\end{array}$ \\
\hline Expressed by the following organisms: \\
\hline 291516108 & Alistipes shahii WAL 8301 \\
291516108 & Alistipes shahii WAL 8301 \\
60495220 & Bacteroides fragilis ATCC 25285 = NCTC 93 \\
291526581 & Eubacterium rectale DSM 17629 \\
291526581 & Eubacterium rectale DSM 17629 \\
295102938 & Faecalibacterium prausnitzii L2/6 \\
148552872 & Methanobrevibacter smithii ATCC 35061; PS; DSMZ \\
324314063 & Odoribacter splanchnicus DSM 220712 \\
294473972 & Prevotella ruminicola Bryant 23 \\
291541371 & Roseburia intestinalis XB6B4 \\
\hline
\end{tabular}

Table 5. Phosphoserine transaminase expression ("control" unique).

\begin{tabular}{|c|c|c|}
\hline 2.6.1.52 & $\begin{array}{l}\text { Phosphoserine } \\
\text { Transaminase }\end{array}$ & $\begin{array}{l}\text { (1) } O \text {-Phospho-L-serine }+ \text { 2-Oxoglutarate }=3 \text {-Phosphonooxypyruvate }+ \text { L-Glutamate } \\
\text { (2) 4-Phosphonooxy-L-threonine }+ \text { 2-Oxoglutarate }= \\
\text { (3R)-3-Hydroxy-2-oxo-4-phosphonooxybutanoate }+ \text { L-Glutamate }\end{array}$ \\
\hline \multicolumn{3}{|c|}{ Expressed by the following organisms: } \\
\hline 167660682 & \multicolumn{2}{|c|}{ Alistipes putredinis DSM 17216} \\
\hline 291516108 & \multicolumn{2}{|c|}{ Alistipes shahii WAL 8301} \\
\hline 60495220 & \multicolumn{2}{|c|}{ Bacteroides fragilis ATCC $25285=$ NCTC 93} \\
\hline 295102938 & \multicolumn{2}{|c|}{ Faecalibacterium prausnitzii L2/6 } \\
\hline 324314063 & \multicolumn{2}{|c|}{ Odoribacter splanchnicus DSM 220712} \\
\hline 294473972 & \multicolumn{2}{|c|}{ Prevotella ruminicola Bryant 23} \\
\hline 291541371 & \multicolumn{2}{|c|}{ Roseburia intestinalis XB6B4 } \\
\hline 291543183 & \multicolumn{2}{|c|}{ Ruminococcus bromii L2-63 } \\
\hline
\end{tabular}


Table 6. 2-oxoglutarate synthase expression ("control” unique).

\begin{tabular}{|c|c|c|}
\hline 1.2.7.3 & 2-Oxoglutarate Synthase & $\begin{array}{l}\text { 2-Oxoglutarate }+\mathrm{CoA}+2 \text { Oxidized Ferredoxin }= \\
\text { Succinyl-CoA }+\mathrm{CO}(2)+2 \text { Reduced Ferredoxin }+2 \mathrm{H}(+)\end{array}$ \\
\hline \multicolumn{3}{|c|}{ Expressed by the following organisms (multiple gene copies present): } \\
\hline 291516108 & \multicolumn{2}{|c|}{ Alistipes shahii WAL $8301(\times 5)$} \\
\hline 148552872 & \multicolumn{2}{|c|}{ Methanobrevibacter smithii ATCC 35061; PS; DSMZ $(\times 5)$} \\
\hline 324314063 & \multicolumn{2}{|c|}{ Odoribacter splanchnicus DSM $220712(\times 3)$} \\
\hline
\end{tabular}

Table 7. Glutamate synthase expression ("control" unique).

\begin{tabular}{|c|c|c|}
\hline 1.4.1.14 & Glutamate Synthase (NADH) & $\begin{array}{l}2 \text { L-Glutamate }+ \text { NAD }(+)= \\
\text { L-Glutamine + 2-Oxoglutarate + NADH }\end{array}$ \\
\hline \multicolumn{3}{|c|}{ Expressed by the following organisms: } \\
\hline 291526581 & \multicolumn{2}{|c|}{ Eubacterium rectale DSM $17629(\times 3)$} \\
\hline 295102938 & \multicolumn{2}{|c|}{ Faecalibacterium prausnitzii L2/6 $(\times 4)$} \\
\hline 291541371 & \multicolumn{2}{|c|}{ Roseburia intestinalis XB6B4 $(\times 3)$} \\
\hline 291543183 & \multicolumn{2}{|l|}{ Ruminococcus bromii L2-63 (×3) } \\
\hline
\end{tabular}

There were several enzymes of interest coded by Lactobacillus acidophilus alone, many involving methionine and cysteine metabolism. Arginase produces ornithine from arginine, a precursor to glutamate. Mercury (II) reductase is included, because sulfur metabolism is involved with detoxifying toxic heavy metals, such as mercury. NADH peroxidase is an antioxidant, and the enzymes associated with methionine metabolism are ultimately associated with glutathione formation, another antioxidant. See Table 8 for these results.

Table 8. Enzymes of interest coded by Lactobacillus acidophilus: Query 1.

\begin{tabular}{|c|c|c|}
\hline 2.1.1.10 & Homocysteine $S$-methyltransferase. & $S$-methyl-L-methionine + L-homocysteine $=2$ L-methionine. \\
\hline 2.1.1.14 & $\begin{array}{l}\text { 5-methyltetrahydropteroyltriglutamate } \\
\text { homocysteine } S \text {-methyltransferase. }\end{array}$ & $\begin{array}{l}\text { 5-methyltetrahydropteroyltri-L-glutamate }+ \text { L-homocysteine }= \\
\text { tetrahydropteroyltri-L-glutamate }+ \text { L-methionine. }\end{array}$ \\
\hline 3.5.3.1 & Arginase. & L-arginine $+\mathrm{H}_{2} \mathrm{O}=$ L-ornithine + urea. \\
\hline 4.2.1.22 & Cystathionine beta-synthase. & L-serine + L-homocysteine $=$ L-cystathionine $+\mathrm{H}_{2} \mathrm{O}$. \\
\hline 4.4.1.1 & Cystathionine gamma-lyase. & L-cystathionine $+\mathrm{H}_{2} \mathrm{O}=$ L-cysteine $+\mathrm{NH}_{3}+$ 2-oxobutanoate. \\
\hline 1.16.1.1 & Mercury(II) reductase. & $\mathrm{Hg}+\mathrm{NADP}^{+}+\mathrm{H}^{+}=\mathrm{Hg}^{2+}+\mathrm{NADPH}$ \\
\hline 2.1.1.176 & 16S rRNA (cytosine(967)-C(5))-methyltransferase. & $\begin{array}{l}S \text {-adenosyl-L-methionine }+ \text { cytosine }(967) \text { in } 16 \mathrm{~S} \text { rRNA }= \\
S \text {-adenosyl-L-homocysteine }+5 \text {-methylcytosine }(967) \text { in } 16 \mathrm{~S} \text { rRNA. }\end{array}$ \\
\hline 1.11.1.1 & NADH peroxidase. & $\mathrm{NADH}+\mathrm{H}_{2} \mathrm{O}_{2}=\mathrm{NAD}^{+}+2 \mathrm{H}_{2} \mathrm{O}$ \\
\hline
\end{tabular}

Other enzymes of interest were antioxidants superoxide reductase (1.15.1.2), coded by Faecalibacterium prausnitzii, and glutathione peroxidase (1.11.1.9), coded by Prevotella ruminicola. Several others exhibited metabolic pathways associated with glutamate, cysteine and methionine and a few for some amino acids of less interest, such as serine or histidine.

The following (Table 9) are some enzymes of interest from the "autism" microbiome. Interestingly, Clostridium perfringens is the only organism studied (among both microbiomes) that codes for glutamate: cysteine ligase and glutathione synthase, the two enzymes needed for glutathione formation. 
Table 9. Enzymes of interest coded by the "autism" microbiome; Query 1.

\begin{tabular}{|c|c|c|c|c|}
\hline 110676061 & Clostridium perfringens ATCC 13124 & 4.1.1.50 & $\begin{array}{l}\text { Adenosylmethionine } \\
\text { decarboxylase. }\end{array}$ & $\begin{array}{l}S \text {-adenosyl-L-methionine }= \\
S \text {-adenosyl 3-(methylthio)propylamine }+\mathrm{CO}_{2} .\end{array}$ \\
\hline 115252745 & Peptoclostridium difficile 630 & 4.1.1.50 & $\begin{array}{l}\text { Adenosylmethionine } \\
\text { decarboxylase. }\end{array}$ & $\begin{array}{l}S \text {-adenosyl-L-methionine }= \\
S \text {-adenosyl 3-(methylthio)propylamine }+\mathrm{CO}_{2} \text {. }\end{array}$ \\
\hline 110676061 & Clostridium perfringens ATCC 13124 & 4.1.1.22 & Histidine decarboxylase. & L-histidine $=$ histamine $+\mathrm{CO}_{2}$. \\
\hline 110676061 & Clostridium perfringens ATCC 13124 & 6.3.2.2 & Glutamate-cysteine ligase. & $\begin{array}{l}\text { ATP }+ \text { L-glutamate }+ \text { L-cysteine }=\text { ADP }+ \\
\text { phosphate + gamma-L-glutamyl-L-cysteine. }\end{array}$ \\
\hline 110676061 & Clostridium perfringens ATCC 13124 & 6.3.2.3 & Glutathione synthase. & $\begin{array}{l}\text { ATP + gamma-L-glutamyl-L-cysteine }+ \\
\text { glycine = ADP + phosphate + glutathione. }\end{array}$ \\
\hline 219869941 & $\begin{array}{l}\text { Desulfovibrio desulfuricans } \\
\text { ATCC } 27774\end{array}$ & 2.6.1.44 & $\begin{array}{l}\text { Alanine-glyoxylate } \\
\text { transaminase. }\end{array}$ & L-alanine + glyoxylate $=$ pyruvate + glycine. \\
\hline 219869941 & $\begin{array}{l}\text { Desulfovibrio desulfuricans } \\
\text { ATCC } 27774\end{array}$ & 1.8 .99 .3 & Hydrogen sulfite reductase. & $\begin{array}{l}\left(\mathrm{O}_{3} \mathrm{~S} . \mathrm{S} \mathrm{SO}_{3}\right)^{2-}+\text { acceptor }+2 \mathrm{H}_{2} \mathrm{O}+\mathrm{OH}^{-}= \\
3 \mathrm{HSO}_{3}{ }^{-}+\text {reduced acceptor. }\end{array}$ \\
\hline 115252745 & Peptoclostridium difficile 630 & 4.4.1.11 & Methionine gamma-lyase. & $\begin{array}{l}\text { L-methionine }+\mathrm{H}_{2} \mathrm{O}= \\
\text { methanethiol }+\mathrm{NH}_{3}+\text { 2-oxobutanoate. }\end{array}$ \\
\hline 115252745 & Peptoclostridium difficile 630 & 1.8.1.2 & Sulfite reductase (NADPH). & $\mathrm{H}_{2} \mathrm{~S}+3 \mathrm{NADP}^{+}+3 \mathrm{H}_{2} \mathrm{O}=$ sulfite $+3 \mathrm{NADPH}$. \\
\hline 115252745 & Peptoclostridium difficile 630 & 5.4 .3 .5 & D-ornithine 4,5-aminomutase. & D-ornithine $=(2 R, 4 S)$-2,4-diaminopentanoate. \\
\hline 115252745 & Peptoclostridium difficile 630 & 5.1 .1 .12 & Ornithine racemase. & L-ornithine $=$ D-ornithine. \\
\hline
\end{tabular}

It was found that Desulfovibrio desulfuricans and Clostridium difficile uniquely coded for enzymes that involved the use of thioredoxins, including sarcosine reductase, betaine reductase and glycine reductase.

Queries 2 and 3 had much less novel results in comparison. We will look at these results first, then take a look at why adding new EC number annotations to the files did not change much in terms of the overall results.

Query 2 only resulted in one new meaningful result (Table 10): the conversion of $S$-adenosyl-methionine to $S$-adenosyl-homocysteine, part of the transsulfuration pathways.

Table 10. SAM ( $S$-adenosyl-methionine) conversion to SAH ( $S$-adenosyl-homocysteine).

\begin{tabular}{lllll}
\hline \multirow{2}{*}{219869941} & Desulfovibrio & Protein-L-isoaspartate & \\
& desulfuricans ATCC 27774 & 2.1 .1 .77 & (D-aspartate) & $S$-adenosyl-L-methionine + protein L-isoaspartate $=$ \\
& & $O$-methyltransferase & $S$-adenosyl-L-homocysteine + protein L-isoaspartate $\alpha$-methyl ester. \\
\hline
\end{tabular}

Query 3 also resulted in only one major meaningful result, as well as two more that are at least noteworthy, involved with cyanide metabolism (Table 11). Of course, cyanide is highly toxic to humans, so the fact that Clostridium difficile is the only organism studied that can metabolize it is interesting, but its relevance to the present study is inconclusive. Also of note was that Bifidobacterium and Lactobacillus of the control microbiome uniquely coded for 2-haloacid dehalogenase, and Lactobacillus uniquely coded for haloalkane dehalogenase. Again, upon searching the literature in regards to these results, the interpretation is inconclusive. The relevance to the present study cannot be determined. 
Table 11. Enzymes of interest coded by the "autism" microbiome in Query 3.

\begin{tabular}{|c|c|c|c|c|}
\hline 115252745 & Peptoclostridium difficile 630 & 2.8.1.2 & $\begin{array}{l}\text { 3-mercaptopyruvate } \\
\text { sulfurtransferase. }\end{array}$ & $\begin{array}{l}\text { 3-mercaptopyruvate }+ \text { cyanide }= \\
\text { pyruvate }+ \text { thiocyanate. }\end{array}$ \\
\hline 115252745 & Peptoclostridium difficile 630 & 2.8.1.1 & Thiosulfate sulfurtransferase. & $\begin{array}{l}\text { Thiosulfate }+ \text { cyanide }= \\
\text { sulfite }+ \text { thiocyanate. }\end{array}$ \\
\hline 115252745 & Peptoclostridium difficile 630 & 4.4.1.8 & Cystathionine beta-lyase. & $\begin{array}{l}\text { L-cystathionine }+\mathrm{H}_{2} \mathrm{O}= \\
\text { L-homocysteine }+\mathrm{NH}_{3}+\text { pyruvate. }\end{array}$ \\
\hline 666001751 & Bifidobacterium longum BXY01 & 3.8.1.9 & (R)-2-haloacid dehalogenase. & $\begin{array}{l}(R) \text {-2-haloacid }+\mathrm{H}_{2} \mathrm{O}= \\
(S) \text {-2-hydroxyacid }+ \text { halide. }\end{array}$ \\
\hline 666001751 & Bifidobacterium longum BXY01 & 3.8.1.2 & (S)-2-haloacid dehalogenase. & $\begin{array}{l}(S) \text {-2-haloacid }+\mathrm{H}_{2} \mathrm{O}= \\
(R) \text {-2-hydroxyacid }+ \text { halide. }\end{array}$ \\
\hline 666001751 & Bifidobacterium longum BXY01 & 3.8 .1 .10 & $\begin{array}{l}\text { 2-haloacid dehalogenase } \\
\text { (configuration-inverting). }\end{array}$ & $\begin{array}{l}\text { (1) }(S) \text {-2-haloacid }+\mathrm{H}_{2} \mathrm{O}= \\
(R) \text {-2-hydroxyacid }+ \text { halide. } \\
(2)(R) \text {-2-haloacid }+\mathrm{H}_{2} \mathrm{O}= \\
(S) \text {-2-hydroxyacid }+ \text { halide. }\end{array}$ \\
\hline 488447870 & Lactobacillus acidophilus La-14 & 3.8.1.2 & (S)-2-haloacid dehalogenase. & $\begin{array}{l}(S) \text {-2-haloacid }+\mathrm{H}_{2} \mathrm{O}= \\
(R) \text {-2-hydroxyacid }+ \text { halide. }\end{array}$ \\
\hline 488447870 & Lactobacillus acidophilus La-14 & 3.8.1.5 & Haloalkane dehalogenase. & $\begin{array}{l}\text { 1-haloalkane }+\mathrm{H}_{2} \mathrm{O}= \\
\text { a primary alcohol }+ \text { halide. }\end{array}$ \\
\hline
\end{tabular}

In order to analyze why some organisms provided more information than others, we compared the number of EC number annotations within each GenBank file to the total CDSs (coding sequences) within each file. For the new organisms added for Queries 2 and 3, we can see that very few annotations were actually added compared to the total CDSs. "Good" coverage seems to lie between 12\% and 25\% when taking the ratio of EC number annotations to total CDSs, and all five of the newly annotated files fall well short of this mark. However, Bifidobacterium had greatly improved coverage after updating EC number annotations, rising from $<4 \%$ to $>25 \%$. Despite this, very few new results were obtained. Table 12 shows these statistics. This table is included in the Supplemental Material as Table S8 as well. It may seem strange at first that there are so few EC number annotations compared to the number of annotated CDSs, but keep in mind that enzymes are only a fraction of the organisms' proteome (e.g., non-catalytic proteins, such as inter-membrane proteins), and some of the organisms' genes code for non-translated RNA transcripts, such as tRNAs. There also seems to be a dearth in annotation of EC numbers in general. 
Table 12. GenBank file statistic comparisons between the number of EC number annotations and coding sequences (CDSs).

\begin{tabular}{|c|c|c|c|c|c|c|c|c|}
\hline \multirow[b]{2}{*}{ Organism Name } & \multicolumn{3}{|l|}{ Original File } & \multicolumn{5}{|c|}{ Updated EC Annotations } \\
\hline & Total CDSs & $\begin{array}{l}\text { Total EC } \\
\text { Numbers }\end{array}$ & $\%$ & $\begin{array}{l}\text { Total EC } \\
\text { Numbers }\end{array}$ & $\begin{array}{l}\text { Total } \\
\text { "Multi-EC" } \\
\text { Designations }\end{array}$ & $\begin{array}{l}\text { Actual } \\
\text { EC Total }\end{array}$ & $\%$ & $\%$ Increase \\
\hline Akkermansia muciniphila & 2138 & 286 & 13.38 & 401 & 34 & 367 & 17.17 & 28.32 \\
\hline Alistipes putredinis & 659 & 92 & 13.96 & 110 & 6 & 104 & 15.78 & 13.04 \\
\hline Alistipes shahii & 2563 & 548 & 21.38 & 603 & 17 & 586 & 22.86 & 6.93 \\
\hline Bacillus subtilis & 4140 & 912 & 22.03 & 941 & 8 & 933 & 22.54 & 2.30 \\
\hline Bacteroides fragilis & 4406 & 366 & 8.31 & 377 & 5 & 372 & 8.443 & 1.64 \\
\hline Bifidobacterium longum & 1903 & 68 & 3.57 & 672 & 189 & 483 & 25.38 & 610.29 \\
\hline Clostridium difficile & 3902 & 1015 & 26.01 & 1,035 & 6 & 1029 & 26.37 & 1.38 \\
\hline Clostridium leptum & 602 & 100 & 16.61 & 114 & 4 & 110 & 18.27 & 10.00 \\
\hline Clostridium perfringens & 2878 & 504 & 17.51 & 539 & 10 & 529 & 18.38 & 4.96 \\
\hline Desulfovibrio desulfuricans & 2356 & 292 & 12.39 & 392 & 32 & 360 & 15.28 & 23.29 \\
\hline Escherichia coli & 4967 & 612 & 12.32 & 1542 & 285 & 1257 & 25.31 & 105.39 \\
\hline Eubacterium rectale & 2898 & 636 & 21.95 & 696 & 16 & 680 & 23.46 & 6.92 \\
\hline Faecalibacterium prausnitzii & 2756 & 586 & 21.26 & 641 & 17 & 624 & 22.64 & 6.48 \\
\hline Lactobacillus acidophilus & 1876 & 486 & 25.91 & 585 & 31 & 554 & 29.53 & 13.99 \\
\hline Methanobrevibacter smithii & 1795 & 414 & 23.06 & 454 & 12 & 442 & 24.62 & 6.76 \\
\hline Odoribacter splanchnicus & 3498 & 515 & 14.72 & 603 & 27 & 576 & 16.47 & 11.84 \\
\hline Prevotella ruminicola & 2791 & 480 & 17.20 & 519 & 12 & 507 & 18.17 & 5.62 \\
\hline Roseburia intestinalis & 3630 & 709 & 19.53 & 793 & 17 & 776 & 21.38 & 9.45 \\
\hline Ruminococcus bromii & 1811 & 467 & 25.79 & 496 & 9 & 487 & 26.89 & 4.28 \\
\hline Weissella koreensis & 1335 & 93 & 6.97 & 288 & 61 & 227 & 17 & 144.09 \\
\hline Bacteroides caccae & 3441 & 0 & 0.00 & 76 & 19 & 57 & 1.656 & \\
\hline Bacteroides thetaiotaomicron & 4787 & 0 & 0.00 & 413 & 125 & 288 & 6.016 & \\
\hline Bacteroides vulgatus & 4065 & 0 & 0.00 & 267 & 80 & 187 & 4.6 & \\
\hline clostridium bolteae & 5830 & 0 & 0.00 & 737 & 227 & 510 & 8.748 & \\
\hline Sutterella wadsworthensis & 2433 & 0 & 0.00 & 136 & 39 & 97 & 3.987 & \\
\hline
\end{tabular}

\section{Discussion}

The present study successfully identified several key enzymes associated with autism spectrum disorders using a bioinformatics data mining approach, by comparing the metabolomes of two distinct microbiomes. We must compare the results to published experimental data in order to evaluate the impact of this study, such as those found in [29-33].

We expected to find key biomarkers unique to Desulfovibrio and Clostridia species indicative of autism, but instead, the key was based on identifying enzymes that were missing from these organisms; hence, a decreased abundance of such enzymes could be used as diagnostic biomarkers for autism. Several different amino acids have previously been reported to be in lower abundance in autistic individuals, but in this study, the amino acid that stood out the most was glutamate. Several organisms in the "control" microbiome coded for enzymes associated with glutamate metabolism, but were curiously missing from the "autism" microbiome. Glutamate is an integral part of glutathione, 
a tripeptide made of glutamate, cysteine and glycine. It was known before that glutathione was down-regulated in autism, but researchers focused their attention on an inhibition of cysteine metabolism to be the culprit.

Upon closer inspection of glutamate's association with autism, we find that there is a hypothesis that does not involve oxidative stress at all. Glutamate is the human body's major excitatory neurotransmitter, which works in opposition to gamma-aminobutyric acid (GABA) [34,35]. Contrary to the Ming et al. study [29], these studies, as well as Adams et al. [31] reported higher quantities of glutamate vs. neurotypical controls. Tevarst van Elst et al. [34] actually reviewed two different hypotheses: that a hypoglutamatergic condition is related to autism and also that a hyperglutamatergic condition is related to autism. Regardless of which one it is, the authors theorize that an imbalance in the neurological excitation/inhibition chemical signaling in the central nervous system is thought to be associated with autism. Furthermore, glutamate decarboxylase is the enzyme responsible for converting glutamate into GABA. Loss of the gene coding for GABA in host neurons has been shown to lead to symptoms characteristic of autism [36]. Going back to the results of our software (i.e., Query 2), we found that glutamate decarboxylase is expressed by organisms from both the control and autism groups. Therefore, what then is the association of our research to the hypotheses on glutamate and autism? That remains to be determined, but we must not ignore the fact that we might not have heard of this field of research had not our software pointed in that direction. Even with limited data availability, it seems that our software can be useful in developing new hypotheses and revealing literature that was previously unknown to us.

After completing our analysis, we came across another study where Akkermansia muciniphila was found in decreased amounts in individuals with autism [37]. According to the authors, Akkermansia is integral to the host's gut health; the gut mucus layer is reduced as Akkermansia composition within the gut is depleted. This contradicts the Finegold et al. pyrosequencing study. In light of these two conflicting studies, it seems that we may have been correct in assuming that the involvement of Akkermansia in autism is questionable at best. We still included it in the study, because theoretically, it should serve as a basis for comparison. If we think that certain Clostridia species and sulfate reducers are indicative of autism, then we may be biased towards results that agree with that assumption. If we include a questionable case, such as Akkermansia, in the "autism" group analysis, then we may better be able to rule out false positives. Regarding this inclusion, no significant results were found in Queries 1 or 2, leading to the exclusion of Akkermansia from Query 3. Query 3 had quite similar results as Queries 1 and 2, which lends credence to the idea that those organisms still included in the "autism" group for Query 3 are more likely to be involved in autism pathogenesis.

Hydrogen sulfide has been cited as a toxic metabolite produced by Desulfovibrio species [10], but data on this metabolite were inconclusive. It was found that Desulfovibrio is not the only organism (in either microbiome) that can metabolize hydrogen sulfide. What we did notice is that Clostridium difficile codes for enzymes that metabolize alternative pathways for ornithine and methionine, precursors to glutamate and cysteine, respectively, with the enzymes ornithine racemase, D-ornithine 4,5-aminomutase, adenosylmethionine decarboxylase and methionine gamma-lyase. Nearly every organism studied codes for enzymes using ferredoxins and thioredoxins, alternative antioxidants to glutathione, which also use a sulfhydryl electron acceptor for reducing power. It is possible that thioredoxins were found in much higher abundance in autistic individuals [28], because thioredoxins had to take over from glutathione as the body's dominant antioxidant. However, if glutathione is usually dominant, then there must be 
something not as chemically favorable associated with ferredoxins and thioredoxins or perhaps they are just not sufficient to fill in the antioxidative gap that glutathione usually fills. That Clostridium difficile can uniquely use ornithine and methionine for other purposes other than glutathione formation provides evidence for this hypothesis. Interestingly, the final two catalytic steps in glutathione formation are uniquely coded by Clostridium perfringens, part of the "autism" microbiome. However, many of the precursor steps are uniquely coded by Lactobacillus acidophilus, part of the "control" microbiome. Glutathione is still existent in autistic individuals, so perhaps Clostridium perfringens allows for some extra utility in glutathione formation; or maybe this is just coincidence.

Quite remarkable was the fact that nearly every reaction of the transsulfuration and transmethylation metabolic pathways was coded by the organisms studied, but that each microbiome ("control" and "autism") could not express the pathways in their entirety alone. This suggests some sort of interdependence between the two microbiomes on completing these pathways. The control microbiome supplies glutamate metabolism, and cysteine and glutathione formation is split between the two groups of organisms. Based on this information, it seems that organisms, such as abnormal Clostridia species and Desulfovibrio, may be products of the sulfur metabolic deficiency found in so many autistic individuals and, therefore, may be essential in filling a unique metabolic niche. However, because of the known toxic byproducts of these organisms, they may still be causing gastrointestinal inflammation and subsequent neuro-inflammation associated with "leaky gut syndrome", leading to regressive autism [15,31]. Therefore, even though these organisms may be causing some major ill effects, the matter becomes complicated by the necessity of their presence in the host. The sulfur metabolic deficiencies and lack of proper anti-oxidation therefore seem to lie at the heart of the problems that lead to regressive autism. If treatment is possible, it would therefore be more likely to entail dietary supplements that restore the metabolic deficiencies to normal conditions [13], rather than eradicating the proposed offending organisms from the host's gut. James et al. [13] reported some improvements in symptoms with specific dietary supplements, and a study by Adams et al. [38] provides some more evidence of this. The Adams et al. study included adult subjects with autism, as well.

It is important to note that there were some crucial limitations to this study. Besides the obvious, that this study is purely computational, the dependence on having annotation data for EC numbers greatly limits the scope of the study. When the first query to the web application was completed and analyzed, it was thought that including more organisms of interest (with newly annotated EC numbers) would greatly strengthen the study. This, in fact, hardly had any impact at all on the initial study. These files had so few annotations, that they did not give good representations of the metabolome of these organisms. Thus, we can only surmise that there may be other more meaningful results possible with better data. It is possible that this study could be improved by creating new gene annotations altogether by using the publically available BLAST tool for sequence alignment of microbial genomic DNA and then annotating these genes with translated gene products and EC numbers. Also of note is that the two Clostridium species studied most deeply in this context (C. perfringens and C. difficile) have not, to our knowledge, been specified as part of the "autism" microbiome, but they happened to be two of the most widely available and comprehensive genomes of Clostridia that could appear in the human gut. Therefore, they were chosen as "representatives" of the Clostridium genus, and thus, associated results should be interpreted with scrutiny. It appears that this type of study has not been attempted previously, yielding mixed results. This provides substantial evidence that the present approach is novel 
and may be a step in a new, potentially useful direction, but its novelty severely limits its impact, because of a lack of resources. It is surmised that as data on microbial genomics and data on gut bacteria associated with autism patients improves, so too will the utility of our novel software and our comparative modeling approach. Future work could include full de novo genomic sequencing of gut microbes and validation through next-generation sequencing devices, followed by comprehensive annotation with accurate EC numbers. On NCBI's server, there is a noted difference in genomic comprehensiveness between microorganisms, where key gut microbes are covered definitively less than model organisms, such as Escherichia coli or Bacillus subtilis. We need to be able to have more studies on gut microbes in their natural habitat and their interactions with host cells in order to have more experimental data to fine-tune our metabolomics model. It also remains to be determined what would be the best way of evaluating data significance from this type of study.

Nevertheless, we still believe that substantial results came about from this study. We have not seen this type of comparative analysis conducted with autism in mind before, and we are proud to say that our computational approach was successful, given some of its parallels to previous experimental studies. Some experimental studies were not able to be validated with this approach, which is to be expected. As stated earlier, this software was designed with the intent to be used for many other applications, besides autism, hence making the software publically available online. Many other human illnesses and conditions are thought to be associated with the human gut microbiome [5], and we believe that these applications might benefit from our approach, as well. Microbiomes in other environments may also benefit; much potential could be lost if the software's use were limited to only the human gut.

\section{Supplemental Materials}

Supplementary materials can be found at http://www.mdpi.com/1422-0067/16/04/8949/s1.

\section{Acknowledgments}

Colin Heberling would like to acknowledge Thomas Koval and the Johns Hopkins Advanced Academic Programs (AAP) graduate program for guidance and for the opportunity to work on this project, as well as Joshua Orvis for instruction in web development as applied to bioinformatics and for his guidance on an early prototype of the online software.

\section{Author Contributions}

Prasad Dhurjati provided a mentoring and supervisory role throughout this project and contributed to its conceptual design. Colin Heberling had a large part in the project's conceptual design and contributed fully to its execution and analysis.

\section{Conflicts of Interest}

The authors declare no conflict of interest. 


\section{References}

1. Baio, J. Prevalence of Autism Spectrum Disorders. In Autism and Developmental Disabilities Monitoring Network, 14 Sites, United States, 2008; Centers for Disease Control and Prevention, Surveillance Summaries: Atlanta, GA, USA, 2012; Volume 61 (SS03), pp. 1-19.

2. CDC Features-Ten Things to Know About New Autism Data. Available online: http://www.cdc.gov/Features/dsAutismData/ (accessed on 25 February 2015).

3. Parracho, H.M.R.T.; Bingham, M.O.; Gibson, G.R.; McCartney, A.L. Differences between the gut microflora of children with autistic spectrum disorders and that of healthy children. J. Med. Microbiol. 2005, 54, 987-991.

4. Adams, J.B.; Johansen, L.J.; Powell, L.D.; Quig, D.; Rubin, R.A. Gastrointestinal flora and gastrointestinal status in children with autism-Comparisons to typical children and correlation with autism severity. BMC Gastroenterol. 2011, 11, 22.

5. Kranich, J.; Maslowski, K.M.; Mackay, C.R. Commensal flora and the regulation of inflammatory and autoimmune responses. Semin. Immunol. 2011, 23, 139-145.

6. Human Microbiome Project Consortium. Structure, function and diversity of the healthy human microbiome. Nature 2012, 486, 207-214.

7. Turnbaugh, P.J.; Hamady, M.; Yatsunenko, T.; Cantarel, B.L.; Duncan, A.; Ley, R.E.; Sogin, M.L.; Jones, W.J.; Roe, B.A.; Affourtit, J.P.; et al. A core gut microbiome in obese and lean twins. Nature 2009, 457, 480-485.

8. Finegold, S.M.; Downes, J.; Summanen, P.H. Microbiology of regressive autism. Anaerobe 2012, $18,260-262$.

9. Finegold, S.M.; Dowd, S.E.; Contcharova, V.; Liu, C.; Henley, K.E.; Wolcott, R.D.; Youn, E.; Summanen, P.H.; Granpeesheh, D.; Dixon, D.; et al. Pyrosequencing study of fecal microflora of autistic and control children. Anaerobe 2010, 16, 444-453.

10. Finegold, S. Desulfovibrio species are potentially important in regressive autism. Med. Hypotheses 2011, 77, 270-274.

11. Wang, L.; Christophersen, C.T.; Sorich, M.J.; Gerber, J.P.; Angley, M.T.; Conlon, M.A. Increased abundance of Sutterlla spp. and Ruminococcus torques in feces of children with autism spectrum disorder. Mol. Autism 2013, 4, 42.

12. Williams, B.L.; Hornig, M.; Buie, T.; Bauman, M.L.; Paik, M.C.; Wick, I.; Bennett, A.; Jabado, O.; Hirschberg, D.L.; Lipkin, W.I. Impaired carbohydrate digestion and transport and mucosal dysbiosis in the intestines of children with autism and gastrointestinal disturbances. PLoS ONE 2011, 6, doi:10.1371/journal.pone.0024585.

13. James, S.J.; Cutler, P.; Melnyk, S.; Jernigan, S.; Janak, L.; Gaylor, D.W.; Neubrander, J.A. Metabolic biomarkers of increased oxidative stress and impaired methylation capacity in children with autism. Am. J. Clin. Nutr. 2004, 80, 1611-1617.

14. Alberti, A.; Pirrone, P.; Elia, M.; Waring, R.H.; Romano, C. Sulphation deficit in "Low-Functioning" autistic children: A pilot study. Biol. Psychiatry 1999, 46, 420-424.

15. Heberling, C.A.; Dhurjati, P.S.; Sasser, M. Hypothesis for a systems connectivity model of autism spectrum disorder pathogenesis: Links to gut bacteria, oxidative stress, and intestinal permeability. Med. Hypotheses 2013, 80, 264-270. 
16. Nealson, K.; Venter, J.C. Metagenomics and the global ocean survey: What's in it for us, and why should we care? ISME J. 2007, 1, 185-190.

17. Rusch, D.B.; Halpern, A.L.; Sutton, G.; Heidelberg, K.B.; Williamson, S.; Yooseph, S.; Wu, D.; Eisen, J.A.; Hoffman, J.M.; Remington, K.; et al. The Sorcerer II Global Ocean sampling expedition: Northwest Atlantic through Eastern Tropical Pacific. PLoS Biol. 2007, 5, doi:10.1371/journal.pbio.0050077.

18. The Human Microbiome Jumpstart Reference Strains Consortium. A catalog of reference genomes from the human microbiome. Science 2010, 328, doi:10.1126/science.1183605.

19. Fernell, E.; Fagerburg, U.L.; Hellström, P.M. No evidence for a clear link between active intestinal inflammation and autism based on analyses faecal calprotectin and rectal nitric oxide. Acta Paediatr. 2007, 96, 1076-1079.

20. Finegold, S.M.; Molitoris, D.; Song, Y.; Liu, C.; Vaisanen, M.L.; Bolte, E.; McTeague, M.; Sandler, R.; Wexler, H.; Marlowe, E.M.; et al. Gastrointestinal microflora studies in late-onset autism. Clin. Infect. Dis. 2002, 35 (Suppl. 1), S6-S16.

21. Heberling, C. Human gut microbiome functional analysis-Exploring the capabilities of the MEGAN Software. April 2013; unpublished.

22. Huson, D.H.; Auch, A.F.; Qi, J.; Schuster, S.C. MEGAN analysis of metagenomic data. Genome Res. 2007, 17, 377-386.

23. Human Microbiome Project Consortium. A framework for human microbiome research. Nature 2012, 486, 215-221.

24. Lin, M.Y.; Chang, F.J. Antioxidative effect of intestinal bacteria Bifidobacterium longum ATCC 15708 and Lactobacillus acidophilus ATCC 4356. Dig. Dis. Sci. 2000, 45, 1617-1622.

25. The National Center for Biotechnology Information; U.S. National Library of Medicine: 8600 Rockville Pike, Bethesda, MD, USA, 2014. Available online: http://www.ncbi.nlm.nih.gov/ (accessed on 25 September 2014).

26. Dhurjati, P.; Mayadevan, R. Systems biology: The synergistic interplay between biology and mathematics. Can. J. Chem. Eng. 2008, 86, 127-141.

27. Heberling, C. Comparative Microbial Metabolomics. Available online: http://cheberling-homepc.com/ comparative_microbial_metabolomics/gene_search_form.cgi (accessed on 12 October 2014).

28. Bairoch, A. The ENZYME database in 2000. Nucleic Acids Res. 2000, 28, 304-305.

29. Ming, X.; Stein, T.P.; Barnes, V.; Rhodes, N.; Guo, L. Metabolic perturbance in autism spectrum disorders: A metabolomics study. J. Proteome Res. 2012, 11, 5856-5862.

30. Al-Yafee, Y.A.; Al-Ayadhi, L.Y.; Haq, S.H.; El-Ansary, A.E. Novel metabolic biomarkers related to sulfurdependent detoxification pathways in autistic patients of Saudi Arabia. BMC Neurol. 2011, $11,139$.

31. Adams, J.B.; Audhya, T.; McDonough-Means, S.; Rubin, R.A.; Quig, D.; Geis, E.; Gehn, E.; Loresto, M.; Mitchell, J.; Atwood, S.; et al. Nutritional and metabolic status of children with autism vs. neurotypical children, and the association with autism severity. Nutr. Metab. 2011, 8, 34.

32. Yap, I.K.S.; Angley, M.; Veselkov, K.A.; Holmes, E.; Lindon, J.C.; Nicholson, J.K. Urinary metabolic phenotyping differentiates children with autism from their unaffected siblings and age-matched controls. J. Proteome Res. 2010, 9, 2996-3004. 
33. Wang, L.; Angley, M.T.; Gerber, J.P.; Sorich, M.J. A review of candidate urinary biomarkers for autism spectrum disorder. Biomarkers 2011, 16, 537-552.

34. Van Tebartz, E.L.; Maier, S.; Fangmeier, T.; Endres, D.; Mueller, G.T.; Nickel, K. Disturbed cingulate glutamate metabolism in adults with high-functioning autism spectrum disorder: Evidence in support of the excitatory/inhibitory imbalance hypothesis. Mol. Psychiatry 2014, 19, 1314-1325.

35. El-Ansary, A.; Al-Ayadhi, L. GABAergic/glutamatergic imbalance relative to excessive neuroinflammation in autism spectrum disorders. J. Neuroinflamm. 2014, 11, doi:10.1186/s12974014-0189-0.

36. Zhang, K.; Hill, K.; Labak, S.; Blatt, G.J.; Soghomonian, J.-J. Loss of glutamic acid decarboxylase (Gad67) in Gpr88-expressing neurons induces learning and social behavior deficits in mice. Neuroscience 2014, 275, 238-247.

37. Wang, L.; Christophersen, C.T.; Sorich, M.J.; Gerber, J.P.; Angley, M.T.; Conlon, M.A. Low relative abundances of the mucolytic bacterium Akkermansia muciniphila and Bifidobacterium spp. in feces of children with autism. Appl. Environ. Microbiol. 2011, 77, 6718-6721.

38. Adams, J.B.; Audhya, T.; McDonough-Means, S.; Rubin, R.A.; Quig, D.; Geis; E.; Gehn, E.; Loresto, M.; Mitchell, J.; Atwood, S.; et al. Effect of a vitamin/mineral supplement on children and adults with autism. BMC Pediatr. 2011, 11, 111.

(C) 2015 by the authors; licensee MDPI, Basel, Switzerland. This article is an open access article distributed under the terms and conditions of the Creative Commons Attribution license (http://creativecommons.org/licenses/by/4.0/). 